\title{
Article
}

\section{Use of pedometers in the workplace as a strategy to increase physical activity}

Harrison, Joanna, O'Donoghue, Annette and Hill, James Edward Available at http://clok.uclan.ac.uk/38320/

Harrison, Joanna ORCID: 0000-0001-8963-7240, O'Donoghue, Annette and Hill, James Edward ORCID: 0000-0003-1430-6927 (2021) Use of pedometers in the workplace as a strategy to increase physical activity. British Journal of Cardiac Nursing . ISSN 1749-6403

It is advisable to refer to the publisher's version if you intend to cite from the work. 10.12968/bjca.2021.0066

For more information about UCLan's research in this area go to

http://www.uclan.ac.uk/researchgroups/ and search for < name of research Group>.

For information about Research generally at UCLan please go to http://www.uclan.ac.uk/research/

All outputs in CLoK are protected by Intellectual Property Rights law, including Copyright law. Copyright, IPR and Moral Rights for the works on this site are retained by the individual authors and/or other copyright owners. Terms and conditions for use of this material are defined in the policies page.

\section{CLoK}

Central Lancashire online Knowledge www.clok.uclan.ac.uk

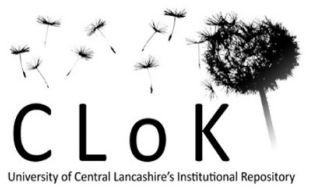


Short Title: The use of pedometers in the workplace to increase physical activity.

\title{
Author Details:
}

Joanna Harrison, Research Fellow ${ }^{(1)}$

Annette O'Donoghue, Clinical Research Therapist ${ }^{(2)}$

James Hill, Senior Research Fellow ${ }^{(1)}$

(1) Synthesis, Economic Evaluation and Decision Science (SEEDS), University of Central Lancashire, Preston

(2) University Hospitals of Morecambe Bay NHS Foundation Trust

Corresponding author: Joanna Harrison, jharrison12@uclan.ac.uk

\begin{abstract}
Lack of physical activity has a negative impact on health outcomes. This is particularly relevant for desk-based workplaces where sitting for long periods is required. This commentary critically appraises and evaluates a Cochrane systematic review of workplace pedometer programmes for increasing physical activity.
\end{abstract}

\section{Acknowledgement}

This report is independent research funded by the National Institute for Health Research Applied Research Collaboration North West Coast (ARC NWC). The views expressed in this publication are those of the author(s) and not necessarily those of the National Institute for Health Research, the NHS, or the Department of Health and Social Care.

\section{Commentary}

A commentary on the following systematic review:

Freak Poli RLA, Cumpston M, Albarqouni L, Clemes SA, Peeters A. 2020.

Workplace pedometer interventions for increasing physical activity. Cochrane Database of 
Systematic Reviews. Issue 7. Art. No.:CD009209.

\section{Key Points}

- Workplace pedometer programmes do not have a definitive impact on physical activity or sedentary behaviour in the medium term.

- Development in step-count technology has now moved onto accelerometers and multi-function activity trackers.

- Future research should explore the effectiveness and sustainability of new technology within health-based workplace programmes.

\section{Introduction}

Sedentary behaviour and physical inactivity are associated with poor health outcomes and unsuccessful aging (Dogra and Stathosokostas 2012; De Rezende et al. 2014). Time spent undertaking sedentary activities such as sitting or lying down is associated with increased risk of cardiovascular disease, type 2 diabetes and all-cause mortality (Wilmot et al. 2012). The associations between sedentary behaviour and all-cause mortality are decreased or effectively removed by regular, moderate to vigorous levels of physical activity (Stamatakis et al. 2019). In the workplace, levels of sedentary behaviour and physical inactivity are high, significantly so in desk workers when compared to manual labour (Castillo-Retamal and Hinckson 2011): Guidance suggests that workplaces support employees to be physically active and encourage the promotion of walking (NICE 2008). Support may be offered via a pedometer, a portable device that gives feedback on step counts and may also increase levels of physical activity (Harris et al. 2017). The Cochrane systematic review by Freak-Poli et 
al. (2020) aimed to ascertain if the use of pedometers in workplace health interventions is effective in increasing physical activity, thereby improving health outcomes.

\section{Methods}

The review undertook a comprehensive literature search from a range of electronic databases including CENTRAL (Cochrane Central Register of Controlled Trials), CINAHL, MEDLINE, Embase, OSH UPDATE, Web of Science, Clinical Trials.gov, and the WHO ICTRP, from inception to December 2016 with an update in 2019. Reference lists from included studies were also searched and searches were not limited by language. Randomised controlled trials of workplace pedometer interventions were included when the pedometer was either the main focus of the intervention or incorporated within a wider package of health promotions. Comparison groups included no or minimal interventions and alternative physical activity interventions.

Screening and data extraction were carried out by two review authors, with arbitration by a third reviewer. Two review authors independently assessed the risk of bias using the Cochrane collaboration tool for risk of bias. The primary outcome assessed was physical activity. The secondary outcomes were sedentary behaviour, cardiovascular disease and type 2 diabetes risk factors, anthropometric measures (e.g. Body Mass Index), blood pressure, biochemical measures (e.g. blood glucose), quality of life and adverse effects including injury. Data was gathered from the longest available period of follow up. Where there was appropriate data, a random effects meta-analysis was undertaken. The quality of the evidence regarding the outcomes was assessed by two authors using the GRADE approach. 


\section{Results}

After screening 6,197 papers, 14 random controlled trials (RCTs) were identified which included 4,762 participants. The 14 RCTs were undertaken in high income countries and were based in a wide range of work placements with a diverse population which were both at risk and not at risk of developing chronic diseases.

When comparing pedometer interventions to no or minimal interventions, there was no strong evidence (very low certainty) of increased physical activity in the medium term $(<1$ year after the programme ended). Similarly, there was no strong evidence (very low certainty) that pedometer interventions improved sedentary behaviour, LDL cholesterol, quality of life: mental health component and systolic blood pressure (moderate certainty evidence). There was some low certainty evidence that pedometer interventions may slightly reduce Body Mass Index in the medium term after the intervention period (MD $-0.64,95 \%$ confidence interval $(\mathrm{Cl})-1.45$ to $0.18, \mathrm{P}=0.12)$ and reduce the risk of adverse events such as injuries at 6 to 9 months (OR $0.50,95 \% \mathrm{Cl} 0.30$ to $0.84, \mathrm{P}=0.009$ ).

When comparing pedometer interventions to alternative physical activity interventions, there was no strong evidence that one of the interventions was superior to the other in increasing physical activity, sedentary time, Body Mass Index, systolic blood pressure and LDL cholesterol (very low certainty evidence).

\section{Commentary}


Exploring the quality of the review using the critical appraisal tool Amstar2 (Shea et al. 2017), this review was identified to be of high quality (scored yes on all 16 criteria) and provides an accurate and comprehensive summary of the results from the included studies. The findings from this review however were limited by a lack of definitive evidence and a small number of studies. The certainty of evidence was downgraded by the review authors due to issues with blinding (participants knowing which study group they were in) and a high risk of participants dropping out of the programme, meaning not enough results were collected. Furthermore, no included studies used pedometers alone as they were all part of broader health promotion interventions. It was therefore difficult to draw conclusions on their effectiveness with other components present. Due to insufficient data being available, it was not possible for the review to explore these different components and other areas of variability such as the age and gender of participants.

Some effects were seen in the medium term ( $<1$ year) but the evidence was of low certainty and findings were not consistent or sustained. Furthermore, when compared to alternative physical activity interventions the overall effect was inconclusive suggesting that pedometerbased interventions do not offer additional benefits for the outcomes assessed within this review. It is therefore not possible to make a recommendation for the widespread use of pedometers to improve health outcomes within the workplace. However, there is evidence elsewhere to suggest positive results for alternative health-based programmes in the workplace. A recent systematic review of reviews identified strong evidence for the effectiveness of such programmes on weight-related outcomes such as Body Mass Index and waist circumference, especially for interventions targeting physical activity and/or diet (10). The same review also identified strong evidence for the prevention of musculoskeletal 
disorders, especially when resistance training was used and a small effect on mental health outcomes, particularly for those interventions that used e-health and cognitive behavioural therapy techniques (Proper KI and van Oostrom 2019).

The review authors acknowledge that the use of pedometers has now been overtaken by accelerometers within smart phones, watches and activity trackers, often at little or no cost to the user and with greater flexibility (e.g. waterproof). Accelerometers are electronic, wearable devices that measure activity counts (accelerations due to body movement) and give electronic feedback to the user. They can also monitor the intensity of physical activity and sedentary behaviour, by measuring activity counts in a specific time period (Migueles et al. 2017). A recent review of consumer based wearable activity trackers identified that they generally resulted in increased physical activity, however this was based on low quality evidence (Brickwood et al. 2019). One of the most common wireless activity trackers on the market is the Fitbit (Diaz et al. 2015), frequently worn as a smartwatch. A review of Fitbit interventions found a significant increase in daily step count, moderate to vigorous physical activity and a significant decrease in weight (Ringeval et al. 2020).

Future research in this area should explore the effectiveness of accelerometers and activity trackers within health-based workplace programmes. Other useful outcomes to explore would be cost-effectiveness, reasons for non-compliance and processes to increase engagement for long-term sustainability.

\section{CPD Reflective Questions}


- What factors would help to make a health-based workplace programme successful in improving physical activity?

- Can issues of participant blinding be overcome in trials of accelerometers and activity trackers?

- What are the main limitations of this systematic review and what would you need to consider when applying the evidence to practice?

\section{References}

Brickwood KJ, Watson G, O'Brien J, Williams AD. 2019. Consumer-Based Wearable Activity Trackers Increase Physical Activity Participation: Systematic Review and Meta-Analysis. JMIR Mhealth Uhealth. 7(4):e11819.

Castillo-Retamal M, Hinckson EA. 2011. Measuring physical activity and sedentary behaviour at work: a review. Work. 40(4):345-57.

De Rezende LF, Rodrigues Lopes M, Rey-López JP, Matsudo VK, Luiz Odo C. 2014. Sedentary behavior and health outcomes: an overview of systematic reviews. PLoS One. 9(8):e105620.

Diaz KM, Krupka DJ, Chang MJ, Peacock J, Ma Y, Goldsmith J, Schwartz JE, Davidson KW. 2015. Fitbit $^{\circledR}$ : An accurate and reliable device for wireless physical activity tracking. Int J Cardiol. 15(185):138-40. 
Dogra S, Stathosokostas L. 2012. Sedentary behaviour and physical activity are independent predictors of successful aging in middle-aged and older adults. J Aging Res. 2012(190654).

Freak-Poli RLA, Cumpston M, Albarqouni L, Clemes SA, Peeters A. 2020.

Workplace pedometer interventions for increasing physical activity. Cochrane Database of Systematic Reviews. Issue 7(Art. No.:CD009209).

Harris T, Kerry SM, Limb ES, Victor CR, Iliffe S, Ussher M, et al. 2017. Effect of a Primary Care Walking Intervention with and without Nurse Support on Physical Activity Levels in 45- to 75-Year-Olds: The Pedometer And Consultation Evaluation (PACE-UP) Cluster Randomised Clinical Trial. PLoS Med 14(1): e1002210.

Migueles JH, Cadenas-Sanchez C, Ekelund U, Delisle Nyström C, Mora-Gonzalez J, Löf M, Labayen I, Ruiz JR, Ortega FB. 2017. Accelerometer Data Collection and Processing Criteria to Assess Physical Activity and Other Outcomes: A Systematic Review and Practical Considerations. Sports Med. 47(9):1821-1845.

NICE. 2008. Physical activity in the workplace. Public Health Guideline [Internet][cited 2021 May 14]. Available from https://www.nice.org.uk/guidance/ph13

Proper KI, van Oostrom SH. 2019. The effectiveness of workplace health promotion interventions on physical and mental health outcomes - a systematic review of reviews. Scand J Work Environ Health. 1;45(6):546-559. 
Ringeval M, Wagner G, Denford J, Paré G, Kitsiou S. 2020. Fitbit-Based Interventions for Healthy Lifestyle Outcomes: Systematic Review and Meta-Analysis. J Med Internet Res. 22(10):e23954.

Shea BJ, Reeves BC, Wells G, Thuku M, Hamel C, Moran J, Moher D, Tugwell P, Welch V, Kristjansson E, Henry DA. 2017. AMSTAR 2: a critical appraisal tool for systematic reviews that include randomised or non-randomised studies of healthcare interventions, or both. BMJ. 21(358):j4008.

Stamatakis E, Gale J, Bauman A, Ekelund U, Hamer M, Ding D. 2019. Sitting Time, Physical Activity, and Risk of Mortality in Adults. J Am Coll Cardiol. 73(16):2062-2072.

Wilmot EG, Edwardson CL, Achana FA, Davies MJ, Gorely T, Gray L, Khunti K, Yates T, Biddle SJ. 2012. Sedentary time in adults and the association with diabetes, cardiovascular disease and death: systematic review and meta-analysis. Diabetologia. 55(11):2895-905. 